\title{
The effect of sensory input on the reaction time of schizophrenic patients of good and poor premorbid history'
}

\author{
Andrew Crider, Brendan Maher 2 and Lester Grinspoon \\ HARVARD UNIVERSITY
}

\begin{abstract}
The reaction times of good premorbid and poor premorbid schizophrenic patients and normal controls were measured under conditions of low, medium and high sensory input (noise). Poor premorbid subjects showed significant initial deficits that improved under conditions of increasing input, reaching normal levels under high input. These findings are congruent with previous research reporting significant biological and behavioral differences between process and reactive schizophrenics. They also clarify the previous findings of improvement in response efficiency reported for schizophrenics generally under conditions of sensory input.
\end{abstract}

\section{Problem}

Simple sensory input (background noise) has been found to improve the performance of withdrawn patients in a reaction-time task (Tizard \& Venables, 1957), of schizophrenic patients in complex discrimination tasks (Pascal \& Swensen, 1952) and in concept formation performance (Cavanaugh, 1958). Improvement of this kind is presumed to be mediated by the changes in arousal state brought about by the sensory input. Differences in autonomic balance between schizophrenic patients with good and poor premorbid histories have been reported by King (1958), De Vault (1955) and Venables (1964), as well as various investigators in the Soviet Union (cf. Lynn, 1963). On this basis, it is to be expected that response improvement under conditions of arousing sensory input will be most marked in those patients showing low sympathetic tone in the resting state-those with a history of poor premorbid adjustment -while the effects upon patients with a good premorbid history should be less striking and closer to those found with normal controls. This is the problem of interest in the present investigation.

\section{Method}

The Ss were 20 patients from the wards of the Massachusetts Mental Health Center bearing unequivocal diagnoses of schizophrenia. These were rated on the Phillips Premorbid History Scale, the ten highest scorers being classified as good premorbid and the ten lower being labelled poor premorbid. Ten male college students served as normal controls. The mean ages of the good and poor premorbid groups were 24.0 and 25.6 years respectively. The controls' mean age was 20.9 years. The difference between these means reached significance (Link-Wallace Method of Allowances, $\mathrm{p}<.05$ ) for the control vs. poor premorbid comparison.
The $\mathrm{S}$ sat in a chair on the arm of which was placed a box holding a $1 \times 3$ in manipulandum. Presentation of a stimulus tone activated a timer which was terminated by depression of the manipulandum. Reaction times were read to units of $1 / 100$ th sec. Tones were varied so as to produce different values of sensory input by varying the intensity of the tone in $\mathrm{db}$ and the interval between presentation of the tones. These parameters were combined to produce one low input condition, two medium input conditions and one high input condition as follows: low input, $75 \mathrm{db} 15 \mathrm{sec}$. interval; medium input, (a) $75 \mathrm{db} 5 \mathrm{sec}$. intervals and (b) $95 \mathrm{db} 15$ sec. intervals; and high input, $95 \mathrm{db} 5$ sec. intervals. Room noise was measured at $57 \mathrm{db}$ throughout the experiment. Each tone series was introduced by a $30 \mathrm{sec}$. warning light and a $3 \mathrm{~min}$. rest period separated each of the four series. Tones were generated by an audio frequency oscillator set at $600 \mathrm{cps}$, relayed through an 8-in speaker placed behind and above the S's chair. One of the 24 possible orders of the four conditions was assigned randomly to each with the restriction that no obvious overbalancing of sequence occurred in any of the three basic subject groups.

\section{Results}

Because the presentation of tone series of this kind produced long latency reactions and startle responses in most Ss, the response to the first tone in each condition was discarded. Mean reaction times were computed for all other responses made in a series. Data obtained for the two medium conditions were compared and the three $t$-values computed for the difference scores between the series taken across the total Ss in each group. In no case did these reach minimal significance. ${ }^{3}$ Therefore each $\mathrm{S}$ 's mean reaction time for the two series was averaged, leaving three conditions distinctly ordered in terms of sensory input.

The data for all three conditions were used in a group $\mathrm{x}$ conditions analysis of variance for correlated observations. Table 1 presents this analysis.

Although the effect for groups is significant, only the differences between the good premorbid and poor premorbid and the normal and poor premorbid groups are reliable. Good premorbids do not differ overall from the normals (Scheffe's Method).

The conditions effect is also significant, and all three comparisons are reliable (Scheffe's Method). The performance of all groups is improved with increasing levels of sensory input. 


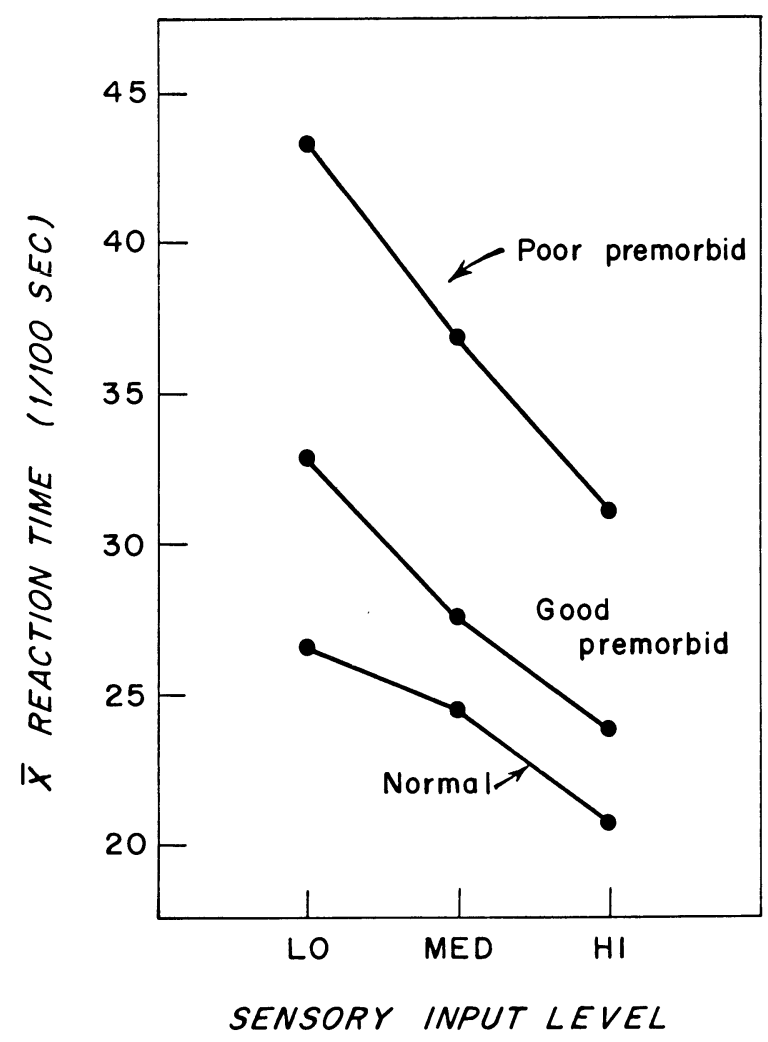

Fig. 1. Reaction time as a function of sensory input level.

The significant interaction indicates that performance improvement is differential for the three groups or, conversely, that group differences are lessened as a function of increasing sensory input (Fig. 1). Since none of the three condition differences between good premorbid and normal groups reaches significance (Link-Wallace Method), the interaction is primarily attributable to the relatively greater improvement over conditions of the poor premorbids. The performance level of this group in the high sensory input condition does not differ significantly from that of the controls in the low input condition (Link-Wallace Method).

\section{Diseussion}

It seems clear that the good premorbid/poor premorbid distinction is related to differences in resting levels of arousal and hence of susceptibility to increased arousal under conditions of sensory input. It is evident also that increasing arousal is accompanied by improved behavioral efficiency insofar as this is measured by
Tavle 1. Ana:ysis of Variance of Mean Reaction Times

\begin{tabular}{lrrr} 
Source & df & MS & \multicolumn{1}{c}{ F } \\
Groups & 2 & 1354.07 & $9.14 * *$ \\
$\quad$ Erro: (a) & 27 & 148.14 & \\
Conditions & 2 & 591.85 & $119.57 * *$ \\
Groups x Conditions & 4 & 28.43 & $5.74 *$ \\
$\quad$ Error (b) & 54 & 4.95 & \\
$* * \mathrm{p}<.001$ & & & \\
$* \mathrm{p}<.01$ & & &
\end{tabular}

faster reaction times. Good premorbid schizophrenics are generally indistinguishable from normal controls in their performance in this situation. These findings thus serve to confirm and extend the growing body of evidence for the existence of significant biological differences between schizophrenic patients where there are established differences in their premorbid social functioning. They also point up the necessity of taking the variables of premorbid adjustment and biological responsiveness into account in any investigation which compares groups of patients diagnosed grossly as "schizophrenic" with other kinds of psychopathology or with normal subjects.

\section{References}

CAVANAUGH, D. K. Improvement in the performance of schizophrenics on concept formation tasks as a function of motivational change. J. abnorm. soc. Psychol., 1958, 57, 8-12.

DeVAULT, S. Physiological responsiveness in reactive and process schizophrenia. Unpublished doctoral dissertation, Michigan State Univer., 1955.

KING, G. F. Differential autonomic responsiveness in the classification of schizophrenia. J. abnorm. soc. Psychol., 1958, 56, 160-164.

LYNN, R. Russian theory and research on schizophrenia. Psychol. Bull, 1963, 60, 486-498.

PASCAL, S., \& SWENSEN, C. Learning in mentally ill patients under conditions of unusual motivation. J. Pers., 1952, 21, 240-249.

TIZARD, J., \& VENABLES, P. The influence of extraneous stimulation on the reaction time of schizophrenics. Brit. J. Psychol., 1957, 48, 299-305.

VENABLES, P. Input dysfunction in schizophrenia. In B. A. Maher (Ed.), Progress in experimental personality research. Vol. I. New York: Academic Press, 1964. Pp. 1-46.

\section{Notes}

1. This study was conducted at the Clinical Research Center of the Massachusetts Mental Health Center, Boston, to which reprint requests should be directed. Supported through NIMH Grants MH-05077 and MPM-19,111. We thank Mr. Bernard Tursky and Dr. Lawrence Gustafson for their assistance during the conduct of this research. 2. Now at the University of Wisconsin.

3. An analysis of variance for intensity and presentation frequency over all 30 Ss showed both main effects to be highly significant $(p<.001)$ and non-interacting. 